\title{
Manifestações fonoaudiológicas relatadas por pais de crianças com hipotireoidismo congênito
}

\section{Speech-language pathology manifestations reported by parents of children with congenital hypothyroidism}

\author{
Ligia Oliva Ferreira ${ }^{1}$, Ranielly da Costa Relvas ${ }^{1}$, Márcia Suely Souza de Castro Ramalho ${ }^{1}$, Claudia Maria Sedrez \\ Gonzaga Ronchi ${ }^{1}$, Viviane Castro de Araújo Perillo ${ }^{1}$, Lidiane Cristina Barraviera Rodrigues ${ }^{1}$
}

\begin{abstract}
RESUMO
Objetivo: Identificar manifestações fonoaudiológicas apresentadas por crianças com hipotireoidismo congênito e investigar a associação de tais manifestações com a época do diagnóstico e o início do tratamento. Métodos: Responsáveis por 15 crianças diagnosticadas com hipotireoidismo congênito e atendidas pelo Serviço de Referência em Triagem Neonatal dos estados de Rondônia e Acre responderam a um questionário no qual deveriam relatar principalmente queixas fonoaudiológicas. A idade das crianças variou de 2 a 7 anos, $67 \%$ de meninos e 33\% de meninas. A média de idade da época do diagnóstico foi de 1 mês e 3 dias e o tratamento iniciou-se, em média, com 1 mês e 6 dias. A dosagem do medicamento variou entre $5 \mathrm{mg} / \mathrm{dia}$ e $100 \mathrm{mg} / \mathrm{dia}$. Resultados: As queixas relatadas foram: atraso para início da linguagem oral, trocas na fala, fala ininteligível, comportamento agitado e comportamento nervoso. Alguns responsáveis não referiram queixas. Verificou-se que a maioria dos sujeitos apresentou problemas na fala (80\%) e a menor parte $(7,7 \%)$, alteração auditiva. Para aqueles que referiram queixa, a média da época do diagnóstico e do início do tratamento foi mais tardia quando comparado àqueles que não as relataram. Conclusão: Foram relatadas manifestações fonoaudiológicas, principalmente relacionadas à fala. Além disso, houve associação entre a presença de queixa e problema de fala com a época do diagnóstico e o início do tratamento. Sugere-se a inserção do fonoaudiólogo no acompanhamento das crianças com hipotireoidismo congênito.
\end{abstract}

Descritores: Hipotireoidismo congênito; Desenvolvimento da linguagem; Desenvolvimento infantil; Cognição; Triagem neonatal; Transtornos desenvolvimento da linguagem/diagnóstico; Transtornos desenvolvimento da linguagem/terapia; Questionários

\section{INTRODUÇÃO}

O hipotireoidismo congênito (HC) é um distúrbio do metabolismo sistêmico caracterizado pela produção deficiente dos hormônios tireoidianos devido ao mau funcionamento ou malformação da glândula tireoide. É a principal causa de retardo mental com possibilidade de ser prevenida se diagnosticada e tratada precocemente ${ }^{(1-3)}$.

O HC é o mais frequente entre as doenças metabólicas, com aproximadamente um caso para quatro mil nascimentos ${ }^{(4,5)}$.

São consideradas causas do HC, distúrbios do desenvolvimento da tireoide, como aplasia da tireoide (atireoidismo); displasia da tireoide; utilização deficiente do iodo; resistência ao hormônio da tireoide; deficiência de TSH e hipotireoidis-

Trabalho realizado na Clínica NATIVIDA, Serviço de Referência em Triagem Neonatal dos Estados de Rondônia e Acre - SRTN - Porto Velho (RO), Brasil. (1) Curso de Graduação em Fonoaudiologia, Faculdade São Lucas - FSL Rondônia (RO), Brasil.

Endereço para correspondência: Lidiane Cristina Barraviera Rodrigues. Av. dos Imigrantes, 5850/704, Bloco A, Aponiã, Porto Velho (RO), Brasil, CEP: 78908-280. E-mail: lidiane@saolucas.edu.br Recebido em: 2/3/2010; Aceito em: 12/8/2010 mo intra-uterino, adquirido por excesso ou carência de iodo durante a gravide ${ }^{(4)}$.

Os hormônios tireoideanos são essenciais para o desenvolvimento do sistema nervoso central e a deficiência desses hormônios na vida fetal e no recém-nascido mantém a imaturidade deste tecido, leva à hipoplasia dos neurônios corticais, gera atraso na mielinização e redução da vascularização. Se a reposição hormonal não for iniciada logo após o nascimento, essas lesões se tornarão irreversíveis, com prejuízo ao desenvolvimento neuropsicomotor ${ }^{(6)}$ podendo levar a alterações de linguagem.

A triagem neonatal (TN), também conhecida como teste do pezinho, permite a detecção precoce do $\mathrm{HC}$ entre outras alterações congênitas do metabolismo ${ }^{(7)}$, o que garante a qualidade de vida da criança acometida ${ }^{(8)}$.

Estudos revelam a importância da intervenção de equipe interdisciplinar para o tratamento de determinadas doenças infantis como distúrbios relacionados à área fonoaudiológica, e os ganhos com impacto na qualidade de vida desses pacientes ${ }^{(9)}$ Entre esses profissionais, merece destaque, por sua importância, a atuação fonoaudiológica nos programas de triagem neonatal ${ }^{(10)}$. 
Nos estados de Rondônia e Acre o Serviço de Referência em Triagem Neonatal (SRTN) é o NATIVIDA, Núcleo de Atendimento em Triagem Neonatal, cadastrado pelo Ministério da Saúde para realizar a coleta do teste do pezinho nestes dois estados, abrangendo 95\% dos recém-nascidos vivos do estado de Rondônia e $80 \%$ do estado do Acre. O mesmo conta com uma equipe multidisciplinar (endocrinologista, pediatra, hematologista, psicólogo, assistente social e nutricionista) que realiza diagnóstico e tratamento de indivíduos com alteração nos resultados da triagem neonatal.

Segundo a literatura ${ }^{(11)}$ os recém-nascidos classificados como suspeitos para o $\mathrm{HC}$ devem ser acompanhados de perto pelos programas de triagem para evitar consequências tardias da doença.

O tratamento consiste de administração de hormônios da tireoide; a dosagem e planejamento terapêutico dependem do grau de deficiência hormonal ${ }^{(4)}$.

Crianças tratadas precocemente terão bom prognóstico mental, com nível cognitivo preservado, resguardadas de repercussões adversas que o $\mathrm{HC}$ pode causar ${ }^{(12)}$.

Poucos estudos no Brasil descrevem as alterações relacionadas aos aspectos fonoaudiológicos e o conhecimento dos pais quanto a essas alterações ${ }^{(10,13)}$. Por isso, o objetivo geral deste estudo foi identificar as manifestações fonoaudiológicas relatadas pelos responsáveis por crianças com HC. Os objetivos específicos foram verificar quantas dessas crianças apresentam manifestações fonoaudiológicas e se há associação entre presença de manifestações fonoaudiológicas em crianças com $\mathrm{HC}$, a época do diagnóstico e o início do tratamento.

\section{MÉTODOS}

Esta pesquisa foi iniciada após a aprovação pelo Comitê de Ética em Pesquisa da Faculdade São Lucas, sob o processo n ${ }^{\circ}$ : 357/2009 seguindo-se as recomendações da resolução 196/96 do Conselho Nacional de Saúde.

O presente estudo foi realizado no Serviço de Referência em Triagem Neonatal dos estados de Rondônia e Acre (SRTN), NATIVIDA, único Serviço de Referência em Triagem Neonatal autorizado pelo SUS para a realização da triagem neonatal, abrangendo os estados de Rondônia e Acre.

Os critérios de inclusão para a participação na pesquisa foram: possuir diagnóstico de hipotireoidismo congênito (HC), apresentar idade entre 2 e 7 anos, realizar tratamento medicamentoso e acompanhamento para o HC no SRTN NATIVIDA - na cidade de Porto Velho (RO).

Foram excluídas da amostra crianças com idade inferior a 2 anos e superior a 7 anos e crianças que apresentassem outras alterações genéticas ou neurológicas diagnosticadas, as quais não fazem parte do quadro do HC, pois as manifestações fonoaudiológicas encontradas podem não ser exclusivamente provocadas pelo $\mathrm{HC}$.

Excluiu-se da amostra um sujeito com diagnóstico de encefalopatia crônica não evolutiva da infância.

A casuística deste estudo foi composta pelos responsáveis por 15 crianças com hipotireoidismo congênito, com idades entre 2 e 7 anos, dez do gênero masculino e cinco do feminino.

A Tabela 1 evidencia a média da época do diagnóstico, início do tratamento e da dosagem do medicamento.

Os participantes foram informados sobre a realização da pesquisa por meio da leitura da Carta de Informação ao Sujeito da Pesquisa e mediante a sua autorização, firmou-se o consentimento assinando o Termo de Consentimento Livre e Esclarecido. Os mesmos responderam a um questionário elaborado pelas pesquisadoras, baseado na literatura ${ }^{(13)}$, composto por perguntas abertas e fechadas sobre os seguintes aspectos: dados pessoais; dados do hipotireoidismo congênito; queixas fonoaudiológicas; período gestacional; período pós-natal; medicamentos; saúde geral; desenvolvimento motor e de linguagem; desempenho social; relação familiar; entre outros.

O questionário foi aplicado pelas pesquisadoras, no próprio Serviço de Referência em Triagem Neonatal dos estados de Rondônia e Acre (SRTN) - NATIVIDA, em Porto Velho (RO), nas datas em que as crianças compareceram para acompanhamento médico, no momento em que estavam aguardando atendimento, em sala anexa.

Para a análise dos dados, foram utilizados os seguintes testes estatísticos: Teste de Igualdade de Duas Proporções, para comparar a proporção de respostas de duas determinadas variáveis e/ou seus níveis; e o Teste de Mann-Whitney, a fim de comparar as queixas e problemas de fala e a época do diagnóstico e o início do tratamento. Foi definido para este estudo o nível de significância estatística de 0,05.

\section{RESULTADOS}

Entre as queixas relatadas pelos responsáveis por crianças com $\mathrm{HC}$ verificamos que quatro indivíduos apresentaram atraso para início da linguagem oral, três relataram trocas na fala, três não referiram queixas, um referiu fala ininteligível, dois mencionaram atrasos de linguagem e trocas na fala, um indivíduo referiu comportamento agitado e outro comportamento nervoso.

Um dos que não referiram queixas, ao ser questionado sobre alterações de fala, relatou dificuldades de compreensão da criança por pessoas distantes do convívio.

Em relação ao período gestacional, verificou-se que $100 \%$ das mães realizaram pré-natal, e que $40 \%$ delas apresentaram intercorrências durante a gestação (hipotireoidismo materno, hepatite B, anemia, infecções urinárias, quedas e exposições a raio- $\mathrm{X})$.

Tabela 1. Média da época do diagnóstico, início do tratamento e da dosagem do medicamento

\begin{tabular}{lcccccccccc}
\hline Aspectos analisados & Média & Mediana & DP & CV (\%) & Q1 & Q3 & Min & Max & $\mathrm{n}$ & IC \\
\hline Idade do diagnóstico (dias) & 37,87 & 30,0 & 35,50 & 94 & 16,0 & 45,0 & 4 & 120 & 15 & 17,97 \\
Início do tratamento (dias) & 47,33 & 30,0 & 30,11 & 64 & 30,0 & 60,0 & 20 & 120 & 15 & 15,24 \\
Dosagem do medicamento (mg/dia) & 44,11 & 43,8 & 25,28 & 57 & 25,0 & 50,0 & 5 & 100 & 14 & 13,24 \\
\hline
\end{tabular}

Legenda: $\mathrm{DP}=$ desvio padrão; CV = coeficiente de variação; Q1 = primeiro quartil; Q3 = terceiro quartil; Min = mínimo; Max = máximo; IC = intervalo de confiança 
Tabela 2. Presença e ausência de alterações nos aspectos do desenvolvimento de linguagem

\begin{tabular}{|c|c|c|c|c|c|}
\hline \multirow{2}{*}{ Desenvolvimento de linguagem } & \multicolumn{2}{|c|}{ Sim } & \multicolumn{2}{|c|}{ Não } & \multirow{2}{*}{ Valor de $p$} \\
\hline & $\mathrm{n}$ & $\%$ & $\mathrm{n}$ & $\%$ & \\
\hline Problemas na fala e linguagem & 12 & 80,0 & 3 & 20,0 & $0,001^{*}$ \\
\hline Compreende ordens & 14 & 93,3 & 1 & 6,7 & $<0,001^{*}$ \\
\hline
\end{tabular}

*Valores significativos $(p \leq 0,05)$ - Teste de Igualdade de Duas Proporções

Tabela 3. Presença e ausência de alterações no desempenho social

\begin{tabular}{|c|c|c|c|c|c|}
\hline \multirow{2}{*}{ Relação familiar } & \multicolumn{2}{|c|}{ Sim } & \multicolumn{2}{|c|}{ Não } & \multirow{2}{*}{ Valor de $p$} \\
\hline & $\mathrm{n}$ & $\%$ & $\mathrm{n}$ & $\%$ & \\
\hline Relaciona-se bem com os familiares & 15 & 100 & 0 & 0,0 & $<0,001^{*}$ \\
\hline Prefere brincar em grupo & 12 & 80 & 3 & 20 & $0,001^{*}$ \\
\hline Relaciona-se com outras crianças & 14 & 93,3 & 1 & 6,7 & $<0,001^{*}$ \\
\hline Adapta-se bem ao meio & 14 & 93,3 & 1 & 6,7 & $<0,001^{*}$ \\
\hline
\end{tabular}

* Valores significativos $(p \leq 0,05)$ - Teste de Igualdade de Duas Proporções

Quanto ao período pós-natal, dois indivíduos necessitaram de medicação, um deles devido à prematuridade e outro, a intercorrências durante o parto. Da amostra, seis relataram icterícia, porém apenas um necessitou de cuidados médicos.

Em relação ao uso do medicamento, 53,3\% (n=8) relataram que seu filho já ficou sem medicação por alguns dias por motivo de esquecimento ou falta do medicamento.

Nenhum dos aspectos de saúde geral pesquisados no questionário teve presença significativa na amostra estudada: os sujeitos não apresentaram problemas de saúde geral ou visuais, e houve baixa ocorrência reportada de traumatismos e problemas auditivos ( $\mathrm{p}<0,001$ para todas as variáveis). Os responsáveis reportaram maior ocorrência de doenças na infância, entretanto não houve diferença significativa entre os sujeitos que apresentaram resposta positiva ou negativa com relação a esse aspecto $(\mathrm{p}=0,715)$ (Figura 1).

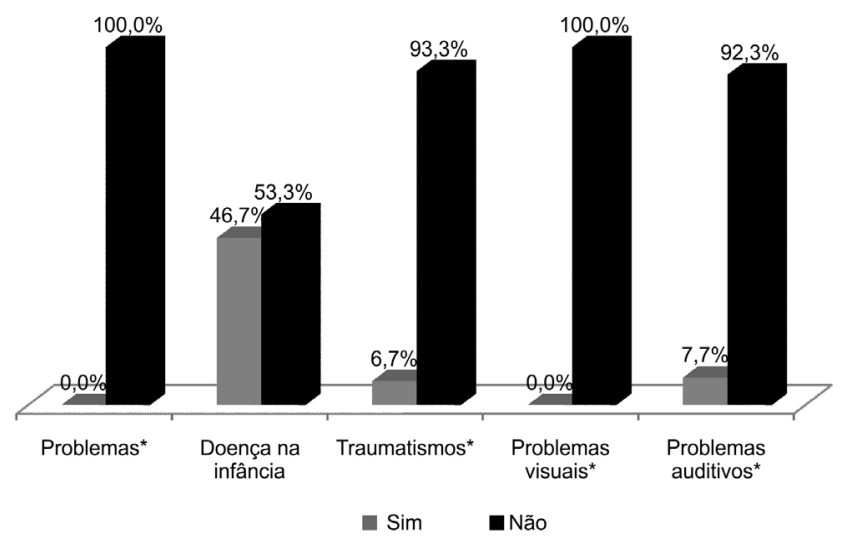

*Valores significativos $(p \leq 0,05)$ - Teste de Igualdade de Duas Proporções

Figura 1. Aspectos de saúde geral

Os resultados quanto aos aspectos do desenvolvimento de linguagem estão apresentados na Tabela 2.

Em relação ao desenvolvimento motor e de linguagem, a média de idade de sustentação de cabeça foi de 6,23 meses (mínimo de 2 meses e máxima de 10 meses); para sentar foi de 8,92 meses (mínimo de 4 meses e máxima de 12 meses); engatinhar 11,64 meses (mínimo de 5 meses e máxima de 24 meses); andar 15,87 meses (mínimo de 8 meses e máxima de 36 meses); balbucio 9,43 meses (mínimo de 5 meses e máxima de 18 meses) e primeiras palavras 17,09 meses (mínimo de 12 meses e máxima de 30 meses).

Quanto ao desempenho social, os resultados estão demonstrados na Tabela 3.

A Tabela 4 demonstra a comparação entre as queixas fonoaudiológicas dos pais com a época do início do diagnóstico e do tratamento. Verifica-se que a diferença entre os resultados não foi significativa para ambas as análises.

Ao comparar os grupos com problemas de fala à época de diagnóstico e do início do tratamento (Tabela 5), verificou-se que não houve diferenças entre eles.

\section{DISCUSSÃO}

$\mathrm{Na}$ presente pesquisa constatou-se maior ocorrência de hipotireoidismo congênito (HC) no gênero masculino. Houve discordância com os resultados de estudo realizado no

Tabela 4. Comparação entre presença e ausência de queixas fonoaudiológicas e época do diagnóstico e início do tratamento

\begin{tabular}{lcccc}
\hline \multirow{2}{*}{ Queixa } & \multicolumn{2}{c}{$\begin{array}{c}\text { Época do diagnóstico } \\
\text { (dias) }\end{array}$} & \multicolumn{2}{c}{$\begin{array}{c}\text { Início do tratamento } \\
\text { (dias) }\end{array}$} \\
\cline { 2 - 5 } & Sim & Não & Sim & Não \\
\hline Média & 43,00 & 17,33 & 50,00 & 36,67 \\
Mediana & 30,0 & 15,0 & 30,0 & 30,0 \\
DP & 37,89 & 11,68 & 32,19 & 20,82 \\
Q1 & 17,8 & 11,0 & 30,0 & 25,0 \\
Q3 & 67,5 & 22,5 & 67,5 & 45,0 \\
n & 12 & 3 & 12 & 3 \\
IC & 21,44 & 13,21 & 18,21 & 23,56 \\
Valor de p & \multicolumn{2}{c}{0,243} & \multicolumn{2}{c}{0,369} \\
\hline
\end{tabular}

Teste de Mann-Whitney $(p \leq 0,05)$

Legenda: DP = desvio-padrão; Q1 = primeiro quartil; Q3 = terceiro quartil; IC = intervalo de confiança 
Tabela 5. Comparação entre presença e ausência de problemas de fala e a época do diagnóstico e início do tratamento

\begin{tabular}{lcccc}
\hline \multirow{2}{*}{$\begin{array}{l}\text { Problema } \\
\text { fala }\end{array}$} & \multicolumn{2}{c}{$\begin{array}{c}\text { Época do diagnóstico } \\
\text { (dias) }\end{array}$} & \multicolumn{2}{c}{ Início do tratamento (dias) } \\
\cline { 2 - 5 } & Sim & Não & Sim & Não \\
\hline Média & 43,00 & 17,33 & 52,50 & 26,67 \\
Mediana & 30,0 & 15,0 & 30,0 & 30,0 \\
DP & 37,89 & 11,68 & 31,66 & 5,77 \\
Q1 & 17,8 & 11,0 & 30,0 & 25,0 \\
Q3 & 67,5 & 22,5 & 67,5 & 30,0 \\
N & 12 & 3 & 12 & 3 \\
IC & 21,44 & 13,21 & 17,91 & 6,53 \\
Valor de $p$ & \multicolumn{2}{c}{0,243} & \multicolumn{2}{c}{0,073} \\
\hline
\end{tabular}

Teste de Mann-Whitney $(\mathrm{p} \leq 0,05)$

Legenda: DP = desvio-padrão; $\mathrm{Q} 1$ = primeiro quartil; $\mathrm{Q} 3$ = terceiro quartil; IC = intervalo de confiança

Pará com o objetivo de determinar o perfil epidemiológico das crianças portadoras de $\mathrm{HC}$, matriculadas no Programa de Triagem Neonatal, no qual 58\% dos acometidos eram do gênero feminino ${ }^{(14)}$. No entanto, concorda com outro estudo, também realizado em Belém- PA, que entrevistou cuidadores de crianças com $\mathrm{HC}$ a fim de identificar variáveis que interferem na adesão ao tratamento ${ }^{(15)}$.

Devemos levar em consideração que as amostras são divergentes, uma vez que o presente estudo possui apenas 15 indivíduos, enquanto o estudo realizado em Belém conta com 131 participantes.

Observou-se que, dos 15 sujeitos, dez iniciaram tratamento antes do primeiro mês de vida, o que está de acordo com a literatura, pois o teste do pezinho deve ocorrer entre o terceiro e sétimo dias de vida, quando a função hormonal do recémnascido já se encontra estabilizada. Este período possibilita o diagnóstico e o início do tratamento precoce dos casos positivos para o HC, que não deve ultrapassar os 30 dias de vida ${ }^{(7)}$.

Com o diagnóstico e tratamento precoce é possível prevenir manifestações e sequelas irreversíveis ${ }^{(4,12,13,16-18)}$.

Verificou-se que todos os sujeitos foram diagnosticados pela triagem neonatal (TN), também conhecida como teste do pezinho, pois de acordo com a literatura, a TN permite a detecção precoce do HC entre outras alterações congênitas do metabolismo ${ }^{(7)}$. Há estudos que ressaltam a importância da sensibilização da população e dos profissionais de saúde em relação à realização da triagem neonatal num prazo máximo de cinco dias de vida da criança ${ }^{(19)}$.

Outros estudos referem que um aspecto importante no cuidado de pacientes com hipotireoidismo congênito detectados por programas de triagem neonatal é o diagnóstico e tratamento precoces para prevenir danos irreversíveis que resultam da falta de tratamento adequado em tempo ideal. A atenção médica imediata poderá prevenir sequelas clínicas sérias, tais como deficiência intelectual, atraso do crescimento, surdez e outras anormalidades do neurodesenvolvimento ${ }^{(18)}$.

Quanto ao uso da medicação verificou-se que todos os sujeitos recebem dosagens de acordo com sua alteração hormonal, e há dúvidas em um caso no qual o responsável não soube informar a dosagem administrada. Segundo pesquisas, a droga é administrada uma vez ao dia, pela manhã. Com o crescimento do corpo, a necessidade hormonal também cresce e exige ajustes de dosagem ${ }^{(4,6,7,20)}$.

Entre as queixas relatadas, destacam-se as relacionadas à fala e linguagem: atraso para início da linguagem oral, trocas e fala ininteligível. Segundo a literatura, em crianças com desempenho alterado nas habilidades do desenvolvimento, observou-se maior déficit nas habilidades de linguagem, principalmente no que diz respeito aos aspectos expressivos e nas habilidades cognitivas ${ }^{(21)}$. Outro estudo com o objetivo de verificar queixas fonoaudiológicas relatadas por pais ou acompanhantes dos pacientes com $\mathrm{HC}$, atendidos pelo Ambulatório Multidisciplinar Especializado da APAE de Anápolis (GO), identificou ocorrência de queixas de atraso para início da linguagem oral ${ }^{(22)}$.

Uma das mães entrevistadas apresentava hipotireoidismo durante a gestação. A criança nasceu com baixo peso. A hipertensão gestacional e o baixo peso fetal são as complicações mais comuns relacionadas ao hipotireoidismo materno. Não é possível afirmar que há diferenças entre o comportamento comunicativo dessa criança e daquelas com hipotireoidismo após o nascimento. Por isso, estudos adicionais são importantes ${ }^{(23)}$.

A literatura refere que o reconhecimento precoce dos aspectos clínicos na situação de deficiência de hormônios tireoidianos tem caráter de emergência pediátrica entre os recém-nascidos. Os sinais mais precoces são, entre outros, icterícia prolongada ou recorrente ${ }^{(6)}$. Na presente pesquisa seis indivíduos referiram icterícia pós-natal, porém apenas um deles necessitou de cuidados hospitalares.

Verificou-se, quanto aos aspectos de saúde geral, uma queixa de alteração auditiva. A literatura refere um estudo com o objetivo de verificar queixas fonoaudiológicas relatadas por pais ou acompanhantes dos pacientes com $\mathrm{HC}$, em que $19 \%$ foram encaminhados para a fonoaudiologia; entre outras queixas, quatro indivíduos apresentaram alterações $\operatorname{auditivas}^{(22)}$.

Observou-se no presente estudo atraso no desenvolvimento motor, o que difere dos relatos da literatura ${ }^{(24)}$. Cabe ressaltar, que para a obtenção de dados desta natureza, ou seja, sobre o início do desenvolvimento dos filhos, os pais deveriam recorrer à memória remota, o que pode ter sido fonte de equívocos. Além disso, pesquisas referem que o hipotireoidismo congênito é um distúrbio metabólico que, quando não tratado precocemente, provoca, além de alterações cognitivas $^{(25)}$ e de linguagem ${ }^{(13)}$, alterações motoras ${ }^{(6,24)}$. A literatura também aponta um relato de caso em que o indivíduo não engatinhou, sentou com um ano e seis meses e andou com dois anos ${ }^{(26)}$.

Quanto à socialização, os resultados deste estudo corroboram a literatura que aponta desempenho adequado para as habilidades sociais ${ }^{(21)}$.

Relacionando-se as queixas dos pais e os problemas de fala relatados com a época do diagnóstico e o início do tratamento, verificou-se que ambos são mais frequentes quando o diagnóstico e o início do tratamento acontecem tardiamente o que está de acordo com afirmações sobre associações entre desempenho adequado nas habilidades motoras, linguísticas, 
cognitivas, sociais e de autocuidados nas crianças que realizaram triagem neonatal (TN), receberam diagnóstico e iniciaram tratamento precoce para o $\mathrm{HC}^{(21)}$.

Há estudos que relatam que crianças tratadas precocemente terão um bom prognóstico intelectual, com nível cognitivo preservado, ficando resguardadas de repercussões adversas que o HC pode causar, como a deficiência mental ${ }^{(11)}$.

Esta pesquisa foi o início de um estudo no Serviço de Referência em Triagem Neonatal dos estados de Rondônia e Acre. Há interesse em confirmar se as queixas relatadas pelos responsáveis por crianças com hipotireoidismo congênito relacionam-se às alterações apresentadas por elas.

\section{CONCLUSÃO}

As manifestações fonoaudiológicas relacionadas às queixas dos responsáveis por crianças com hipotireoidismo congênito foram: atraso para início da linguagem oral, trocas na fala, fala ininteligível e alteração auditiva.

Houve relação entre a presença de queixa e problema de fala com a época do diagnóstico e o início do tratamento.

Diante do exposto, sugere-se a inserção do fonoaudiólogo em programas que atuam com indivíduos com hipotireoidismo congênito, pois o mesmo, ao participar da equipe, poderá contribuir para o desenvolvimento das habilidades comunicativas com caráter preventivo e terapêutico.

\begin{abstract}
Purpose: To identify speech-language pathology manifestations presented by children with congenital hypothyroidism, and to investigate the association of such manifestations with the time of diagnosis and beginning of treatment. Methods: Caregivers of 15 children diagnosed with congenital hypothyroidism who attended the Reference Service on Neonatal Screening of the states of Rondônia and Acre answered a questionnaire in which they should report mainly speech-language pathology complaints. Children's age ranged from 2 to 7 years, $67 \%$ of boys and 33\% of girls. The average age at the time of diagnosis was 1 month and 3 days, and treatment started, in average, at 1 month and 6 days. The dosage of the drug varied from $5 \mathrm{mg} /$ day to $100 \mathrm{mg} /$ day. Results: The complaints reported were: delay in the onset of oral language, speech alterations, unintelligible speech, agitated and nervous behavior. Some caregivers did not report any complaints. It was found that most subjects had problems with speech (80\%), and a few had hearing impairment (7.7\%). For those who reported complaints, mean time of diagnosis and beginning of treatment was delayed when compared to those who did not report. Conclusion: Speech-language pathology manifestations were reported, especially regarding speech. Moreover, there was association between the presence of complaints and speech disorders with the time of diagnosis and beginning of treatment. It is suggested the inclusion of the speech-language pathologist and audiologist in the team monitoring the development of children with congenital hypothyroidism.
\end{abstract}

Keywords: Congenital hypothyroidism; Language development; Child development; Cognition; Neonatal screening; Language development disorders/diagnosis; Language development disorders/therapy; Questionnaires

\title{
REFERÊNCIAS
}

1. Pantoja Ludueña M, Gonzáles de Prada EM, Paulsen Sandi K. Hipotiroidismo congénito: A propósito de un caso. Rev Soc Boliv Pediatr. 2002;41(1):11-4.

2. Silva LP, Elnecave RH. Doenças da tireoide. In: Ferreira JP. Pediatria: diagnóstico e tratamento. Porto Alegre: Artmed; 2005. p. 597-9.

3. González VG, Santucci ZC, Pattin J, Apezteguía M, Borrajo G. Programa de pesquisa neonatal de hipotiroidismo congénito de la provincia de Buenos Aires: 1.377.455 niños evaluados en diez años de experiência. Arch Argent Pediatr. 2007;105(5):390-7.

4. Bottcher T, Engelhardt S, Kortenhaus M. Pediatria de Netter. Porto Alegre: Artmed; 2005. p.336-8.

5. LaFranchi S. Congenital hypothyroidism: etiologies, diagnosis, and management. Thyroid. 1999;9(7):735-40.

6. Setian NS. Hypothyroidism in children: diagnosis and treatment. J Pediatr (Rio J). 2007;83(5 Suppl):S209-16.

7. Brasil. Ministério da Saúde. O passo a passo para a triagem [Internet]. Brasília: Ministério da Saúde; [s.d.] [citado 2011 Jun 14]. Disponível em: http://portal.saude.gov.br/portal/saude/visualizar_texto. cfm?idtxt=24981\&janela $=1$

8. França SN, Domingos MT. Triagem neonatal do hipotireoidismo congênito: novas conquistas novos desafios. Arq Bras Endocrinol Metab. 2008;52(4):579-80.
9. Rabelo BG, Salomão LM, Carnivali PA, Leite IC. Algumas considerações sobre o grau de conhecimento dos pediatras sobre questões fonoaudiológicas. Fono Atual. 2004;7(27):4-10.

10. Gejão MG, Ferreira AT, Lamônica DA. Importância do fonoaudiólogo no acompanhamento de indivíduos com hipotireoidismo congênito. Rev CEFAC. 2008;10(3):287-92.

11. Ramalho AR, Ramalho RJ, Oliveira CR, Santos EG, Oliveira MC, Aguiar-Oliveira MH. Programa de triagem neonatal para hipoteroidismo congênito no nordeste do Brasil: critérios diagnósticos e resultados. Arq Bras Endocrinol Metab. 2008;52(4):617-27.

12. Figueiredo CK, Shermann L. Interação mãe-criança e problemas de comportamento infantil em crianças com hipotireoidismo congênito. Psicol Reflex Crít. 2001;14(3):487-95.

13. Gejão MG. Habilidades do desenvolvimento em crianças com hipotireoidismo congênito: enfoque na comunicação [dissertação]. São Paulo, Bauru: Universidade de São Paulo, Faculdade de Odontologia de Bauru; 2006.

14. Benevides AM, Lima CH, Rocha CA, Corrêa AR, El Husny AS, Fernandes-Caldato MC. Perfil epidemiológico de portadores de hipotireoidismo congênito. Rev Para Med. 2006;20(3):23-6. 
15. Oliveira FP, Ferreira EA. Adesão ao tratamento do hipotireoidismo congênito segundo relato de cuidadores. Psicol Reflex Crít. 2010;23(1):19-28.

16. Nascimento ML, Pires MM, Nassar SM, Ruhland L. Avaliação do programa de rastreamento neonatal para hipotireoidismo congênito da Secretaria de Estado da Saúde de Santa Catarina. Arq Bras Endocrinol Metab. 2003;47(1):75-81.

17. Borges AR, Oliveira LQ,Colares PDT. A importância da triagem neonatal no diagnóstico precoce do hipotireoidismo congênito. Estudos. 2006;33(11/12):925-35.

18. Dantas M, Miguel D, Pereira M, Toralles MB, Alves C. A importância da triagem neonatal no hipotireoidismo congênito: análise secundária de dados. Gazeta Médica da Bahia. 2007;76 (supl 3):S82-S87.

19. Pezzuti IL, Lima PP, Dias VM. Hipotireoidismo congênito: perfil clínico dos recém-nascidos identificados pelo Programa de Triagem Neonatal de Minas Gerais. J Pediatr (Rio J). 2009;85(1):72-9.

20. Subramanian S, Holt AF. Hipotireoidismo. In: Henderson KE, Baranski TJ, Bickel PE. The Washington manual série consultas: endocrinologia. Traduzido por Maria de Fátima Azevedo. Rio de Janeiro: Guanabara Koogan; 2006. p. 47-54.
21. Gejão MG, Lamônica DA. Habilidades do desenvolvimento em crianças com hipotireoidismo congênito: enfoque na comunicação. Pró-Fono. 2008;20(1):25-30.

22. Marinho RT, Costa AA, Machado NC, Ferreira VL, Campos LPD, Gonçalves PBC. Principais queixas fonoaudiológicas de pacientes com hipotireodismo congênito atendidos no ambulatório multidisciplinar especializado da APAE de Anápolis. In: V Congresso Brasileiro de Triagem Neonatal; 2008. Caldas Novas (GO); 2008. p. 34.

23. Smallridge RC. Hypothyroidism and pregnancy. Endocrinologist. 2002;12(5):454-64.

24. Azevedo MF. Avaliação audiológica no primeiro ano de vida. In: Lopes Filho O. Tratado de fonoaudiologia. 2a ed. Ribeirão Preto: Tecmed; 2005. p.235-58.

25. Androvandi C, Nunes ML. Avaliação intelectual de escolares com hipotireoidismo congênito. Aletheia. 2004;(20):55-64.

26. Castilho SD, Costa NA. Diagnóstico precoce do hipotireoidismo congênito: desafio na prática clínica frente a um teste de triagem neonatal normal. Rev Ciênc Med (Campinas). 2007;16(4/6):291-5. 